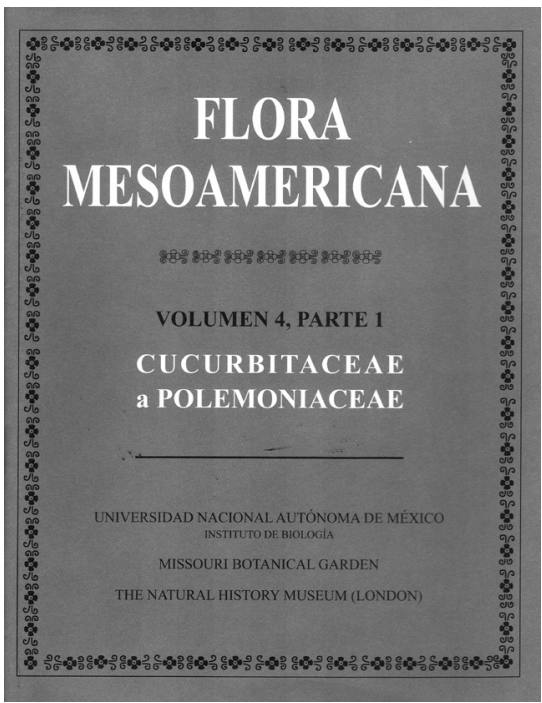

$\mathbf{E}^{1}$ 1 Volumen 4 Parte 1 de la Flora Mesoamericana, publicado en 2009 y ampliamente distribuido en 2010, comprende la revisión taxonómica de 33 familias de angiospermas con 329 géneros y un total de 2,146 especies. Incluye la nomenclatura, las claves de identificación y las descripciones botánicas a nivel de familia, género, especie y taxa infraespecíficos; en forma adicional, se indican los tipos de vegetación y las altitudes donde crecen estas plantas, la fenología, la distribución geográfica, los nombres comunes y los usos de las especies en el ámbito regional o local. El área geográfica definida para la Flora Mesoamericana incluye los estados de Tabasco, Chiapas, Campeche, Quintana Roo y Yucatán en México y los países centroamericanos (Belice, Guatemala, Honduras, El Salvador, Nicaragua, Costa Rica y Panamá).

\title{
De LAS CUCURBITACEAE A LAS POLEMONIACEAE EN LA FLORA MESOAMERICANA: DIVERSIDAD, FITOGEOGRAFÍA Y SISTEMÁTICA DE LOS GÉNEROS Y ESPECIES DE ESTAS fAMILIAS EN MESOAMÉRICA
}

\author{
Davidse, G., Sousa S., M. Knapp, S. y Chiang, F. Eds. \\ Flora Mesoaméricana VOlumen 4, PARTE 1 \\ CUCURBITACEAE A POLEMONIACEAE
}

\author{
Universidad Nacional Autónoma de México, Instituto de Biología; Missouri \\ Botanical Garden; The Natural History Museum (London). 855 páginas. 2009. \\ ISBN 968-36-3309-9 Flora Mesoamericana. \\ ISBN 979-607-02-0901-7 Volumen 4 Parte 1.
}

\section{Diversidad de géneros y especies}

$\mathrm{Al}$ analizar la diversidad de géneros y especies de las 33 familias incluidas en este volumen, destacan la familia Melastomataceae con 477 especies correspondientes a 37 géneros, la familia Apocynaceae [incluyendo a las Asclepiadaceae] con 333 especies distribuidas en 66 géneros, la familia Myrtaceae con 254 especies en 20 géneros, la familia Myrsinaceae con 198 especies en 10 géneros, la familia Ericaceae con 144 especies en 29 géneros y la familia Cucurbitaceae con 114 especies en 33 géneros. En el otro extremo, se encuentran 12 familias representadas en la región por un sólo género, entre ellas, las Plumbaginaceae con dos especies, las Styracaceae con 11, las Ebenaceae con 15 especies, las Clethraceae con 16 y las Symplocaceae con 22 especies.

Una comparación del número de especies por familia en México y en Mesoamérica indica que, en algunos casos, México cuenta con mayor diversidad que Mesoamérica como en las familias Apocynaceae (431 especies en México/333 especies en Mesoamérica), Onagraceae (214/47), Cucurbitaceae (163/114), Lythraceae (120/55), Polemoniaceae (116/15) y Gentianaceae (101/67). En otras familias se presenta lo contrario, hay un mayor número de especies en Mesoamérica que en México; tal es el caso de las Melastomataceae (477 especies en Mesoamérica/212 especies en México), Myrtaceae (254/124), Myrsinaceae (198/129), Ericaceae (144/109), Sapotaceae (90/46), Araliaceae (73/25) y Lecythidaceae (37/1). El número de géneros y especies por familia en México se ha completado con datos registrados por Almeda (1993), Alvarado (2004), Calderón (1996), Carranza (2005), Díaz-Barriga (1993), Gon- 
zález (1993), Graham (1991, 1994), Juárez et al. (2007), Kelly (2001), Lira y Rodríguez (1999), López-Ferrari (1993), Medina-Lemos (1994), Nash (1979), Nee (1993), Pacheco (1981, 1983), Rzedowski y Calderón (1995), Sánchez-Vindas (1990), Sosa y Gómez-Pompa (1994), Todzia (1995), Villarreal $(2001,2008)$ y Villaseñor (2004).

\section{Patrones de distribución geográfica}

A partir de los datos proporcionados en este volumen de la Flora Mesoamericana es posible reconocer los principales patrones de distribución geográfica a nivel genérico y específico.

Para los géneros correspondientes a las 33 familias, los patrones de distribución geográfica son los siguientes: el $31 \%$ de los géneros se distribuyen de México a Sudamérica, un $17 \%$ de los géneros se encuentra en Mesoamérica y Sudamérica, otro $17 \%$ corresponde a géneros pantropicales y cosmopolitas, un $9 \%$ se extiende de Estados Unidos a Sudamérica, otro $9 \%$ se restringe a México y Mesoamérica, $7 \%$ se encuentran desde Estados Unidos a Mesoamérica, otro $7 \%$ son géneros del Viejo Mundo y sólo el 3\% corresponde a géneros endémicos de Mesoamérica.

A nivel específico los patrones de distribución geográfica son distintos: sólo el $10 \%$ de las especies se distribuyen de México a Sudamérica, el $21 \%$ de las especies se encuentra en Mesoamérica y Sudamérica, sólo un $2 \%$ de las especies son pantropicales y cosmopolitas, otro $2 \%$ se extiende de Estados Unidos a Sudamérica, el $14 \%$ de las especies se restringe a México y Mesoamérica, sólo un 2\% se encuentran desde Estados Unidos a Mesoamérica, mientras que el $48 \%$ corresponde a especies endémicas de Mesoamérica. Datos para algunas familias fueron tomados de Steyermark et al. $(1995,1998,1999)$.

El número de especies endémicas de Mesoamérica y endémicas de México y Mesoamérica por familia se resume en el siguiente cuadro:

\begin{tabular}{|c|c|}
\hline $\begin{array}{l}\text { Número de } \\
\text { especies } \\
\text { endémicas de } \\
\text { Mesoamérica }\end{array}$ & $\begin{array}{l}\text { Número de } \\
\text { especies } \\
\text { endémicas } \\
\text { de México y } \\
\text { Mesoamérica }\end{array}$ \\
\hline Melastomataceae (221) & (39) \\
\hline Myrtaceae (163) & (24) \\
\hline Apocynaceae (145) & (63) \\
\hline Myrsinaceae (139) & (25) \\
\hline Ericaceae (89) & (11) \\
\hline Araliaceae (53) & (7) \\
\hline Sapotaceae (35) & (11) \\
\hline Gentianaceae (30) & (9) \\
\hline Cucurbitaceae (26) & (25) \\
\hline Symplocaceae (19) & (2) \\
\hline Apiaceae (17) & (19) \\
\hline Clethraceae (11) & (4) \\
\hline Lecythidaceae (10) & (1) \\
\hline Lythraceae (10) & (22) \\
\hline Teophrastaceae (9) & (4) \\
\hline Ebenaceae (8) & (2) \\
\hline Polemoniaceae (6) & (6) \\
\hline Styracaceae (6) & (4) \\
\hline Loganiaceae (5) & (3) \\
\hline Oleaceae (5) & (5) \\
\hline Onagraceae (3) & (9) \\
\hline Gunneraceae (2) & (1) \\
\hline Combretaceae (1) & (3) \\
\hline Garryaceae (1) & (1) \\
\hline Nyssaceae (1) & $(0)$ \\
\hline Primulaceae (1) & (0) \\
\hline
\end{tabular}

\section{Comentarios editoriales}

La edición del Volumen 4 Parte 1 de la Flora Mesoamericana es excelente. Se manifiesta el trabajo cuidadoso y coordinado de los editores y los traductores con los 59 especialistas participantes, adscritos a 33 instituciones de nueve países (Canadá, Costa Rica, España, Estados Unidos, Holanda, México, Reino Unido, Suecia y Suiza). La traducción al español de numerosos tratamientos taxonómicos, por Jennifer H. Bain, Alejandra Zal- dívar y Juan Pablo Abascal, es de alta calidad.

Esta obra tendrá un gran impacto en el trabajo botánico de muchas regiones de América y es ya una referencia obligada para investigaciones de muy diversa índole, no sólo taxonómicas y florísticas, sino también ecológicas, fitogeográficas, etnobotánicas, filogenéticas y filogeográficas, entre otras. Este volumen de la Flora Mesoamericana constituye una herramienta fundamental para la determinación de ejemplares botánicos de la región, en numerosas colecciones de Herbarios a nivel mundial.

\section{Comentarios finales}

La diversidad de géneros y especies de las 33 familias de plantas con flores incluidas en el Volumen 4 Parte 1 de la Flora Mesoamericana es una muestra de la gran riqueza florística y el alto grado de endemismo de la región. Los proyectos como la Flora Mesoamericana tienen gran impacto en el conocimiento y la conservación de la biodiversidad, en la formación de jóvenes investigadores y en el incremento de las colecciones botánicas. El apoyo decidido de las instituciones editoras y de otras instituciones con especialistas participantes en la Flora Mesoamericana es fundamental para completar esta obra tan relevante.

\section{Literatura citada}

Almeda F. 1993. Melastomataceae. Fascículo 10:1-38. En: Rzedowski J. y Calderón G. Eds. Flora del Bajío y de Regiones Adyacentes. Instituto de Ecología, A.C., Pátzcuaro.

Alvarado L.O. 2004. Apocynaceae. Fascículo 38:1-57. En: Kelly L.M., Ochoterena H. y Medina-Lemos R. Eds. Flora del Valle de Tehuacán-Cuicatlán. Universidad Nacional Autónoma de México. México, D.F.

Calderón G. 1996. Plumbaginaceae. Fascículo 44:1-11. En: Rzedowski J. y Calderón G. Eds. Flora del Bajío y de Regiones Adyacentes. Instituto de Eco- 
logía, A.C., Pátzcuaro.

Carranza E. 2005. Sapotaceae. Fascículo 132:1-28. En: Rzedowski J. y Calderón G. Eds. Flora del Bajío y de Regiones Adyacentes. Instituto de Ecología, A.C., Pátzcuaro.

Díaz-Barriga H. 1993. Symplocaceae. Fascículo 19:1-6. En: Rzedowski J. y Calderón G. Eds. Flora del Bajío y de Regiones Adyacentes. Instituto de Ecología, A.C., Pátzcuaro.

González E. 1993. Styracaceae. Fascículo 21:1-14. En: Rzedowski J. y Calderón G. Eds. Flora del Bajío y de Regiones Adyacentes. Instituto de Ecología, A.C., Pátzcuaro.

Graham S.A. 1991. Lythraceae. Fascículo 66:1-94. En: Sosa V. Ed. Flora de Veracruz. Instituto de Ecología, A.C. y University of California-Riverside, Xalapa.

Graham S.A. 1994. Lythraceae. Fascículo 24:1-62. En: Rzedowski J. y Calderón G. Eds. Flora del Bajío y de Regiones Adyacentes. Instituto de Ecología, A.C., Pátzcuaro.

Juárez V., Alvarado L.O. y Villaseñor J.L. 2007. Apocynaceae, s.1. Revista Mexicana de Biodiversidad 78:459-482.

Kelly L.M. 2001. Ebenaceae. Fascículo 34:1-5. En: Kelly L.M., Ochoterena H. y Medina-Lemos R. Eds. Flora del Valle de Tehuacán-Cuicatlán. Universidad Nacional Autónoma de México, México, D.F.

Lira R. y Rodríguez I. 1999. Cucurbitaceae. Fascículo 22:1-63. En: Dávila P.D., Villaseñor J.L., Medina-Lemos R. y Téllez O. Eds. Flora del Valle de TehuacánCuicatlán. Universidad Nacional Autónoma de México, México, D.F.

López-Ferrari A.R. 1993. Araliaceae. Fascículo 20:1-17. En: Rzedowski J. y Calderón G. Eds. Flora del Bajío y de Regiones Adyacentes. Instituto de Eco- logía, A.C., Pátzcuaro.

Medina-Lemos R. 1994. Araliaceae. Fascículo 4:1-13. En: Dávila P.D., Villaseñor J.L., Medina-Lemos R. y Téllez O. Eds. Flora del Valle de TehuacánCuicatlán. Universidad Nacional Autónoma de México, México, D.F.

Nash D.L. 1979. Polemoniaceae. Fascículo 7:1-27. En: Gómez-Pompa A. Ed. Flora de Veracruz. Instituto Nacional de Investigaciones sobre Recursos Bióticos, Xalapa.

Nee M. 1993. Cucurbitaceae. Fascículo 74:1-133. En: Sosa V. Ed. Flora de Veracruz. Instituto de Ecología, A.C. $\mathrm{y}$ University of California-Riverside, Xalapa.

Pacheco L. 1981. Ebenaceae. Fascículo 16:1-21. En: Gómez-Pompa A. Ed. Flora de Veracruz. Instituto Nacional de Investigaciones sobre Recursos Bióticos, Xalapa.

Pacheco L. 1983. Styracaceae. Fascículo 32:1-12. En: Gómez-Pompa A. Ed. Flora de Veracruz. Instituto Nacional de Investigaciones sobre Recursos Bióticos, Xalapa.

Rzedowski J. y Calderón G. 1995. Polemoniaceae. Fascículo 33:1-41. En: Rzedowski J. y Calderón G. Eds. Flora del Bajío y de Regiones Adyacentes. Instituto de Ecología, A.C., Pátzcuaro.

Sánchez-Vindas P.E. 1990. Myrtaceae. Fascículo 62:1-146. En: Gómez-Pompa A. Ed. Flora de Veracruz. Instituto de Ecología, A.C. y University of California-Riverside, Xalapa.

Sosa V. y Gómez-Pompa A. Comp. 1994. Lista Florística. Fascículo 82:1-245. En: Sosa V. Ed. Flora de Veracruz. Instituto de Ecología, A.C. y University of California-Riverside, Xalapa.

Steyermark J.A., Berry P.E. y Holst B.K. Eds. 1995. Flora of the Venezuelan
Guayana Vol. 1. Missouri Botanical Garden Press. San Luis.

Steyermark J.A., Berry P.E. y Holst B.K. Eds. 1998. Flora of the Venezuelan Guayana Vol. 4 Missouri Botanical Garden Press, San Luis.

Steyermark J.A., Berry P.E. y Holst B.K. Eds. 1999. Flora of the Venezuelan Guayana Vol. 5 Missouri Botanical Garden Press, San Luis.

Todzia C.A. 1995. Melastomataceae. Fascículo 8:1-13. En: Dávila P.D., Villaseñor J.L., Medina-Lemos R. y Téllez O. Eds. Flora del Valle de Tehuacán-Cuicatlán. Universidad Nacional Autónoma de México, México, D.F.

Villarreal J.A. 2001. Gentianaceae. Fascículo 121:1-67. En: Sosa V. Ed. Flora de Veracruz. Instituto de Ecología, A.C. y University of California-Riverside, Xalapa.

Villarreal J.A. 2008. Gentianaceae. Fascículo 60:1-18. En: Medina-Lemos R. Ed. Flora del Valle de Tehuacán-Cuicatlán. Universidad Nacional Autónoma de México, México, D.F.

Villaseñor J.L. 2004. Los géneros de plantas vasculares de la flora de México. Boletín de la Sociedad Botánica de México 75:105-135.
Rosaura Grether
Departamento de Biología,
División de CBS,
Universidad Autónoma
Metropolitana-Iztapalapa
Apartado Postal 55-535,
C.P. 09340, México, D.F.
rogg@xanum.uam.mx 